This is an electronic reprint of the original article. This reprint may differ from the original in pagination and typographic detail.

Author(s): Bazhenov, Andrey; Lefferts, Leon; Honkala, Karoliina

Title: $\quad$ Adsorption and Activation of Water on Cuboctahedral Rhodium and Platinum Nanoparticles

Year: $\quad 2017$

Version:

Please cite the original version:

Bazhenov, A., Lefferts, L., \& Honkala, K. (2017). Adsorption and Activation of Water on Cuboctahedral Rhodium and Platinum Nanoparticles. Journal of Physical Chemistry C, 121(8), 4324-4331. https://doi.org/10.1021/acs.jpcc.6b11953

All material supplied via JYX is protected by copyright and other intellectual property rights, and duplication or sale of all or part of any of the repository collections is not permitted, except that material may be duplicated by you for your research use or educational purposes in electronic or print form. You must obtain permission for any other use. Electronic or print copies may not be offered, whether for sale or otherwise to anyone who is not an authorised user. 


\title{
Adsorption and Activation of Water on Cuboctahedral Rhodium and Platinum Nanoparticles
}

\author{
Andrey S. Bazhenov, Leon Lefferts, and Karoliina Honkala
}

J. Phys. Chem. C, Just Accepted Manuscript • DOI: 10.1021/acs.jpcc.6b11953 • Publication Date (Web): 07 Feb 2017

Downloaded from http://pubs.acs.org on February 10, 2017

\section{Just Accepted}

"Just Accepted" manuscripts have been peer-reviewed and accepted for publication. They are posted online prior to technical editing, formatting for publication and author proofing. The American Chemical Society provides "Just Accepted" as a free service to the research community to expedite the dissemination of scientific material as soon as possible after acceptance. "Just Accepted" manuscripts appear in full in PDF format accompanied by an HTML abstract. "Just Accepted" manuscripts have been fully peer reviewed, but should not be considered the official version of record. They are accessible to all readers and citable by the Digital Object Identifier (DOI®). "Just Accepted" is an optional service offered to authors. Therefore, the "Just Accepted" Web site may not include all articles that will be published in the journal. After a manuscript is technically edited and formatted, it will be removed from the "Just Accepted" Web site and published as an ASAP article. Note that technical editing may introduce minor changes to the manuscript text and/or graphics which could affect content, and all legal disclaimers and ethical guidelines that apply to the journal pertain. ACS cannot be held responsible for errors or consequences arising from the use of information contained in these "Just Accepted" manuscripts. 


\title{
Adsorption and Activation of Water on Cuboctahedral Rhodium and Platinum Nanoparticles
}

\author{
Andrey S. Bazhenov, ${ }^{\dagger}$ Leon Lefferts, ${ }^{\ddagger}$ and Karoliina Honkala*,† \\ $\dagger$ Nanoscience Center, Department of Chemistry, University of Jyväskylä, P.O. Box 35, \\ FI-40014 Jyväskylä, Finland \\ $\ddagger$ Faculty of Science and Technology, University of Twente, P.O. Box 217, 7500 AE \\ Enschede, the Netherlands \\ E-mail: karoliina.honkala@jyu.fi \\ Phone: +35840 8053686
}


$\mathrm{Rh}$ and Pt are widely used as the components in heterogeneous catalysts for multiple industrial applications. Since the metals are typically in the form of nanoparticles in real catalysts, it is important to carefully select models for the computational prediction of the catalytic properties. Here, we report a first-principle study on the water activation, an important step in numerous catalytic reactions, using the finite-size Rh and Pt nanoparticle models and compare them to the extended surface models. We show that regardless of the model, adsorption and activation of water is practically identical for both metals, whereas the dissociation is energetically more favorable on Rh. The experimentally observed difference must be thus attributed to stronger interaction of dissociated water with the metal surfaces and/or to the presence of the oxide support. Through a selection of descriptors, we demonstrate that the extended surface models cannot fully represent the atomic and electronic structures of the small nanoparticles of less than $2 \mathrm{~nm}$ in size.

\section{Introduction}

Heterogeneous transition metal catalysts are widely exploited nowadays in major industrial processes to obtain renewable fuels and sustainable chemicals. In particular, $\mathrm{Rh}$ and $\mathrm{Pt}$ have been found active in the water-gas shift and multiple reforming reactions, as highlighted in the recent reviews. ${ }^{1-6}$ Activation of water has been reported to play a key role in the networks of the aforementioned reactions, in some cases being acknowledged as the rate-limiting step for the water-gas shift cycle. ${ }^{7,8}$ Since the industrial catalysts operate at the nanoscale regime, deeper atomic-level understanding of the water activation in the heterogeneous transition metal-based catalytic systems could provide important information for systematization and rational design of advanced catalysts with higher activity and selectivity.

Along with other transition metals, adsorption and dissociation of water on the surfaces of Rh and Pt have been computationally addressed by numerous research groups during 
the last decade. ${ }^{9-18}$ Calculation reports have been primarily focused on representing metal surfaces with flat and stepped surface slabs to mimic different structural features of realistic nanoparticles at relatively low computational expenses. The flat (111) and (100) surfaces of Rh and Pt have been shown to adsorb and activate water equally well. ${ }^{11}$ The (211) and (221) stepped surfaces, mimicking the edges of realistic nanoparticles, have been found to be more reactive towards water, which is likely due to the lower coordination of the surface sites. ${ }^{10,11}$ These findings are in line with the computational reports by Calle-Vallejo et al. ${ }^{19-21}$ and Fajín et al., ${ }^{12}$ where the authors have demonstrated the stronger tendency of water to bind the undercoordinated edge and corner sites over the facets using the finite-size Pt nanoparticles. Moreover, the degree of interaction has been observed to depend on the size of Pt nanoparticles, ${ }^{12}$ making the extended surface models insufficient in describing the finite-sized facets of small nanoparticles.

To our knowledge, computational studies on water activation by $\mathrm{Pt}$ and, especially, Rh nanoparticles are limited, and the comparison between finite-sized $\mathrm{Rh}$ and $\mathrm{Pt}$ nanoparticles in their tendencies to bind and activate water remains incomplete. Here, we report a firstprinciples study, where we seek answers to the following questions: (1) what is the difference between Rh and Pt in their activity towards adsorption and activation of water, especially, in the nanoscale regime; (2) how well do the extended surfaces represent the finite-size features of small Rh and Pt nanoparticles; and (3) are the electronic structure and surface coordination sufficient descriptors that could determine quantitatively the ability of Rh and Pt metals to bind and activate water?

\section{Computational Details}

Spin-paired periodic and cluster density functional theory (DFT) calculations were performed using the grid-based projected augmented wave approach as implemented in the GPAW. ${ }^{22,23}$ The Perdew-Burke-Ernzerhof (PBE) functional ${ }^{24,25}$ was employed throughout the study. 
The wave-functions were represented on a direct lattice grid with the maximal grid density of $0.20 \AA$. Atomic projected augmented wave (PAW) setups were employed to every atom. The core electrons were treated in a frozen-core approximation in the case of $\mathrm{Rh}, \mathrm{Pt}$, and $\mathrm{O}$ atoms. The PAW setups included 15 valence electrons for Rh, 16 valence electrons for Pt, 6 valence electrons for $\mathrm{O}$, and 1 valence electron for $\mathrm{H}$. The reciprocal space was sampled using the $(6 \times 6 \times 1)$ Morkhorst-Pack-type mesh of $k$-points in the 2D-periodic calculations, while the non-periodic systems were treated at the $\Gamma$-point alone. The optimized lattice constants of $3.857 \AA$ and $3.981 \AA$ were used for the bulk Rh and Pt metals, respectively. Extended surfaces were represented by either $(2 \times 2)$ flat or $(3 \times 2)$ stepped slabs of four atomic layers thick, with the two bottom layers fixed to the original positions. Cuboctahedral nanoparticles were generated via the Wulff's construction as implemented in the ASE code. ${ }^{26}$ The required values for the surface energies were taken from the Crystalium database. ${ }^{27,28}$ Two different size models included 55 and 147 metal atoms, resulting in a set of nanoparticles with the diameter of approximately $1.0-1.7 \mathrm{~nm}$. Only the atoms in the topmost layers were allowed to relax. Complete relaxation of the nanoparticle models results in the changes in the considered energy quantities of no more than $\pm 0.05 \mathrm{eV}$ and may be neglected, thereby reducing the computational burden without significant loss in accuracy. Extended surface models were surrounded by at least $5 \AA$ of vacuum below and $10 \AA$ above the slabs. The nanoparticle models were surrounded by at least $7.5 \AA$ of vacuum in each direction. Each structure was minimized until the maximal residual force was below $0.05 \mathrm{eV} \AA^{-1}$. The transition states of water activation were located using the constraint optimization technique with a $0.025 \AA$ step for the cleaving bond and confirmed by the presence of a single imaginary mode that corresponds to the reaction coordinate. The gas-phase water molecule was calculated in a $12.8 \times 12.8 \times 12.8 \AA$ non-periodic computational cell on a $64 \times 64 \times 64$ points uniform grid (grid spacing $0.20 \AA$ ). The vibrational analysis was thereby performed only for the adsorbate atoms. In the dissociated states, $\mathrm{OH}$ and $\mathrm{H}$ species were separated as far away from each other as possible by placing them either into individual unit cells in the case of the extended 

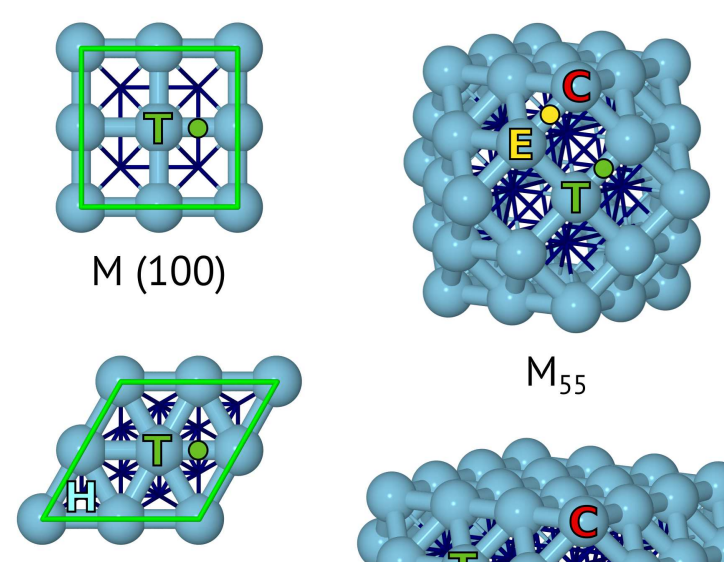

M (111)

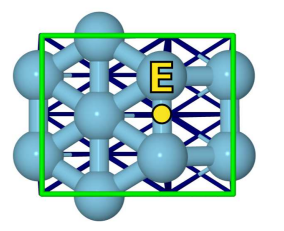

M (211)

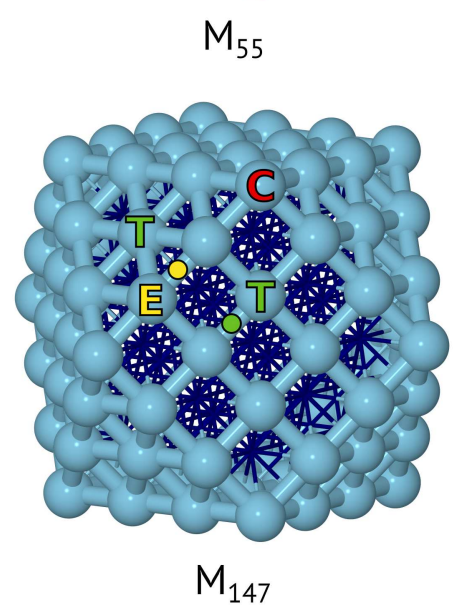

Figure 1: Structural models exploited throughout the study (bulk metal atoms in dark blue, surface metal atoms in light blue, unit cells of the extended surfaces in green). In the designation, $\mathrm{T}$ stands for the terrace, $\mathrm{H}$ for the hollow fcc, $\mathrm{E}$ for the edge, and $\mathrm{C}$ for the corner sites; T-T and E-E stand for the bridging sites (represented by the dots) at the terraces and edges, respectively.

Figure 1 represents all structural models exploited throughout the study with the relevant for discussion surface sites highlighted. The cuboctahedral metal nanoparticles are terminated by two families of facets, namely hexagonal (111) and square (100), which differ in the coordination of metal atoms. The former features 9-coordinated $111 \mathrm{~T}$ sites and can be thus mimicked with the extended (111) surface, whereas the latter features 8-coordinated $100 \mathrm{~T}$ sites and is mimicked with the extended (100) surface. The edges between the (111) and (100) facets consist of 7-coordinated E sites, and we represent those with the step edge 
sites on the extended (211) surface. The nanoparticle edges further intersect to form the $\mathrm{C}$ sites, at which the metal atoms are 5-coordinated. The $\mathrm{C}$ sites can be, in principle, represented by high-index extended surfaces (as shown, for example, in Ref. ${ }^{29}$ ); however, this representation lies beyond the scope of the current study.

Although the surface sites in the extended surfaces have identical coordination numbers as the corresponding sites in the nanoparticles, the $x y$-periodic surfaces lack mimicking finite size effects of the structural motifs in the nanoparticles. To overcome such a misalignment, Calle-Vallejo et al. ${ }^{19}$ suggested a descriptor, the so-called generalized coordination number, $\overline{\mathrm{CN}}$, which is calculated as

$$
\overline{\mathrm{CN}}=\frac{\sum \mathrm{CN}_{i} n_{i}}{\mathrm{CN}_{\max }},
$$

where $n_{i}$ in the number of atoms with the conventional coordination number $\mathrm{CN}_{i}$ located in the first coordination sphere of the surface site of interest and $\mathrm{CN}_{\max }$ is the maximal value of the coordination number in the system, which equals 12 for the considered "atop" sites on the fcc metals. Table 1 gives the generalized coordination numbers for the relevant adsorption sites in the studied systems (Figure 1). The values of $\overline{\mathrm{CN}}$ for the sites in the nanoparticles approach those for the corresponding sites in the extended surfaces with the increasing size of the nanoparticles, making the generalized coordination number a more descriptive quantity, which reflects better the size-dependent nature, as has been successfully shown by the authors in a comprehensive evaluations of the interaction of water and other small species with various sites of truncated octahderal Pt nanoparticles. ${ }^{19-21}$

We evaluated adsorption, activation, and dissociation of molecular water on the sites specified in Figure 1. Water was found to bind each examined site preferably in the "atop" position (Figure 2). The transition states of activation, illustrated in Figure 3, were located at the region of $1.5-1.7 \AA$ for the length of the cleaving $\mathrm{O}-\mathrm{H}$ bond. The adsorption, $\Delta E$, and transition state, $\Delta E^{\ddagger}$, energies (Table 2) were calculated with respect to the infinitely separated species as $\Delta E^{(\ddagger)}=E_{\Sigma}^{(\ddagger)}-\left(E_{\mathrm{S}}+E_{\mathrm{W}}\right)$, where $E_{\Sigma}^{(\ddagger)}, E_{\mathrm{S}}$, and $E_{\mathrm{W}}$ are the DFT energies 
Table 1: Generalized coordination numbers of the relevant sites on the nanoparticles and extended surfaces.

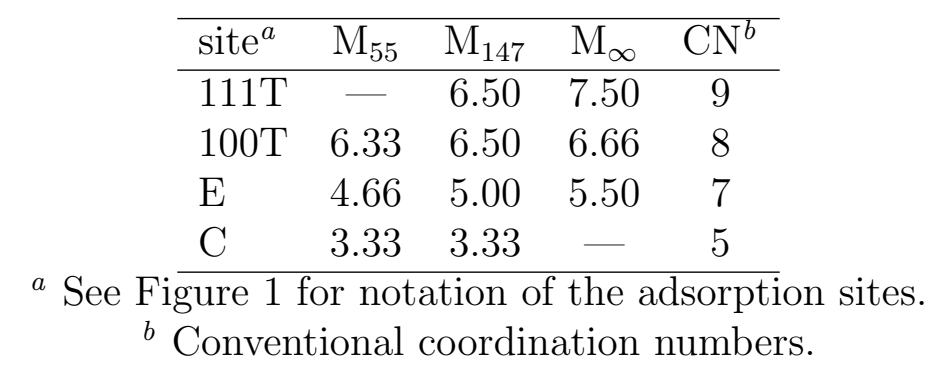

of the metal-water (transition state) complex, plain metal, and gas-phase water molecule, respectively. Table 3 shows the energy of the dissociated state, $\Delta E^{\mathrm{D}}$, which was calculated similarly for the nanoparticles and as $\Delta E^{\mathrm{D}}=\left(E_{\Sigma}^{\mathrm{OH}}+E_{\Sigma}^{\mathrm{H}}\right)-\left(2 E_{\mathrm{S}}+E_{\mathrm{W}}\right)$ in the case of the extended surfaces, where $E_{\Sigma}^{\mathrm{OH}}$ and $E_{\Sigma}^{\mathrm{H}}$ are the respective DFT energies of the metal-OH and metal-H complexes. In such formulation, more negative values indicate stronger interaction and/or greater stability.

Table 2: Adsorption $(\Delta E)$ and transition state $\left(\Delta E^{\ddagger}\right)$ energies (in eV) and corresponding imaginary frequencies $\left(\omega\right.$, in $\left.\mathrm{cm}^{-1}\right)$ for the most favorable configurations of water at the representative sites on $\mathrm{Rh}$ and $\mathrm{Pt}$ nanoparticles and extended surfaces.

\begin{tabular}{llllllll}
\hline site $^{a}$ & model & $\Delta E(\mathrm{Rh})$ & $\Delta E(\mathrm{Pt})$ & $\Delta E^{\ddagger}(\mathrm{Rh})$ & $\omega(\mathrm{Rh})$ & $\Delta E^{\ddagger}(\mathrm{Pt})$ & $\omega(\mathrm{Pt})$ \\
\hline $111 \mathrm{~T}$ & $\mathrm{M}_{\infty}$ & -0.34 & -0.29 & +0.53 & $450.0 i$ & +0.59 & $366.2 i$ \\
& $\mathrm{M}_{147}$ & -0.19 & -0.16 & +0.72 & $610.6 i$ & +0.80 & $368.1 i$ \\
\multirow{2}{*}{$100 \mathrm{~T}$} & $\mathrm{M}_{\infty}$ & -0.34 & -0.25 & +0.55 & $488.3 i$ & +0.55 & $236.6 i$ \\
& $\mathrm{M}_{147}$ & -0.24 & -0.23 & +0.61 & $456.3 i$ & +0.56 & $230.8 i$ \\
& $\mathrm{M}_{55}$ & -0.22 & -0.19 & +0.56 & $428.8 i$ & +0.57 & $324.8 i$ \\
$\mathrm{E}$ & $\mathrm{M}_{\infty}$ & -0.39 & -0.37 & +0.44 & $446.4 i$ & +0.39 & $279.9 i$ \\
& $\mathrm{M}_{147}$ & -0.37 & -0.36 & +0.36 & $500.1 i$ & +0.30 & $499.8 i$ \\
& $\mathrm{M}_{55}$ & -0.38 & -0.34 & +0.42 & $306.0 i$ & +0.41 & $307.7 i$ \\
$\mathrm{C}$ & $\mathrm{M}_{147}$ & -0.56 & -0.66 & +0.26 & $699.2 i$ & +0.19 & $305.7 i$ \\
& $\mathrm{M}_{55}$ & -0.57 & -0.59 & +0.20 & $542.6 i$ & +0.10 & $632.4 i$ \\
\hline \multicolumn{5}{c}{ See Figure 1 for notation of the adsorption sites. }
\end{tabular}

Molecular adsorption of water was found to be practically equivalent (within $0.10 \mathrm{eV}$ ) for the corresponding sites on Rh and Pt. The adsorption energies on the extended surface models indicate that water favors the E site on the stepped (211) surfaces over the terrace sites on the (111) and (100) surfaces in line with the previous computational reports. ${ }^{9-18}$ The 

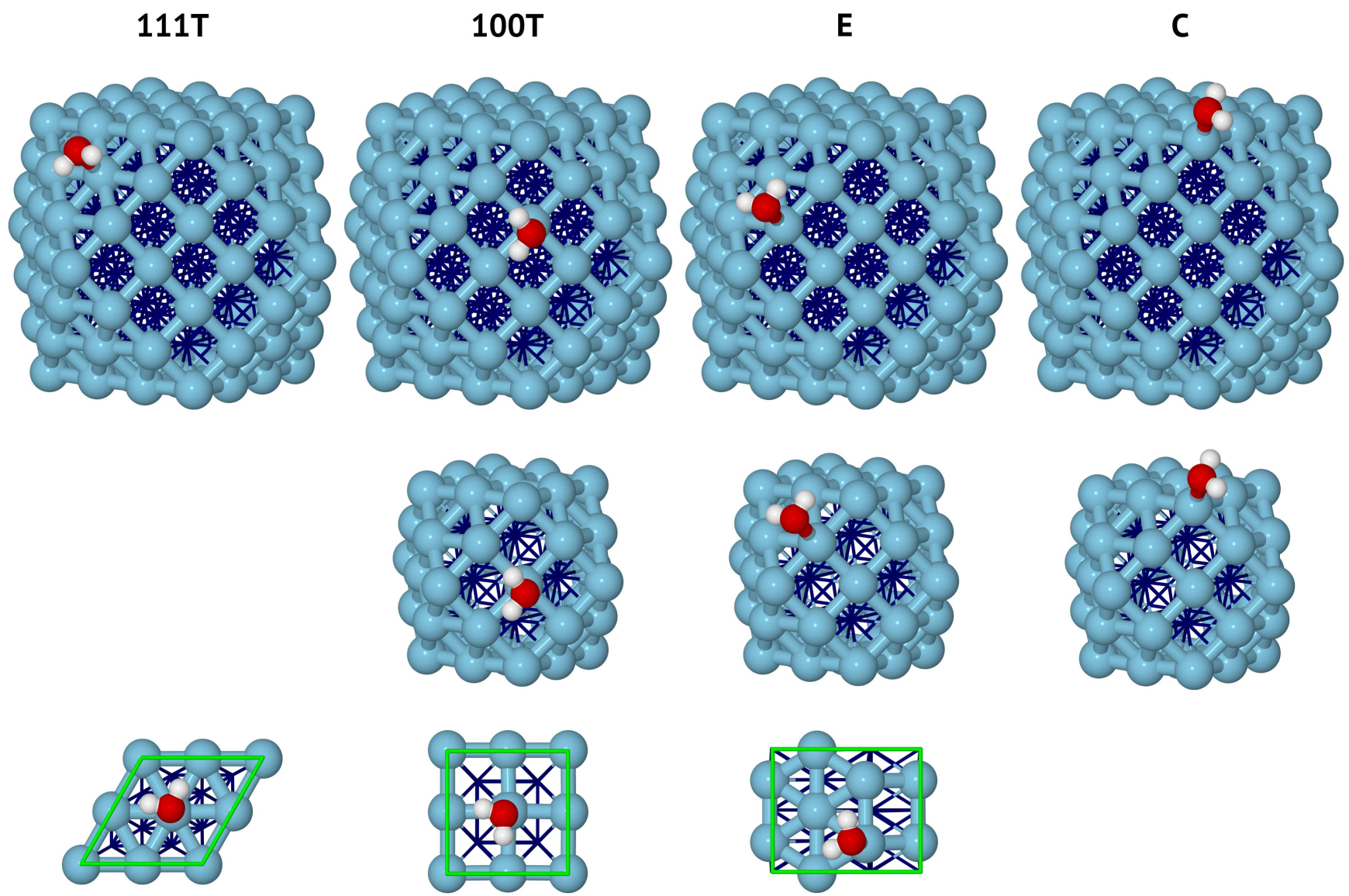

Figure 2: Adsorption modes of molecular water on each studied surface site (bulk metal atoms in dark blue, surface metal atoms in light blue, oxygen in red, hydrogen in white, unit cells of the extended surfaces in green). See Figure 1 for the notation of the surface sites.

Table 3: Dissociated state energies $\left(\Delta E^{\mathrm{D}}\right.$, in $\left.\mathrm{eV}\right)$ for the most favorable configurations of dissociated water on $\mathrm{Rh}$ and $\mathrm{Pt}$ nanoparticles and extended surfaces.

\begin{tabular}{lll}
\hline model & $\Delta E^{\mathrm{D}}(\mathrm{Rh})$ & $\Delta E^{\mathrm{D}}(\mathrm{Pt})$ \\
\hline $\mathrm{M}(111)$ & -0.28 & +0.30 \\
$\mathrm{M}(100)$ & -0.60 & -0.28 \\
$\mathrm{M}(211)$ & -0.82 & -0.52 \\
$\mathrm{M}_{147}$ & -0.93 & -0.73 \\
$\mathrm{M}_{55}$ & -0.73 & -0.66 \\
\hline
\end{tabular}


$111 \mathrm{~T}$
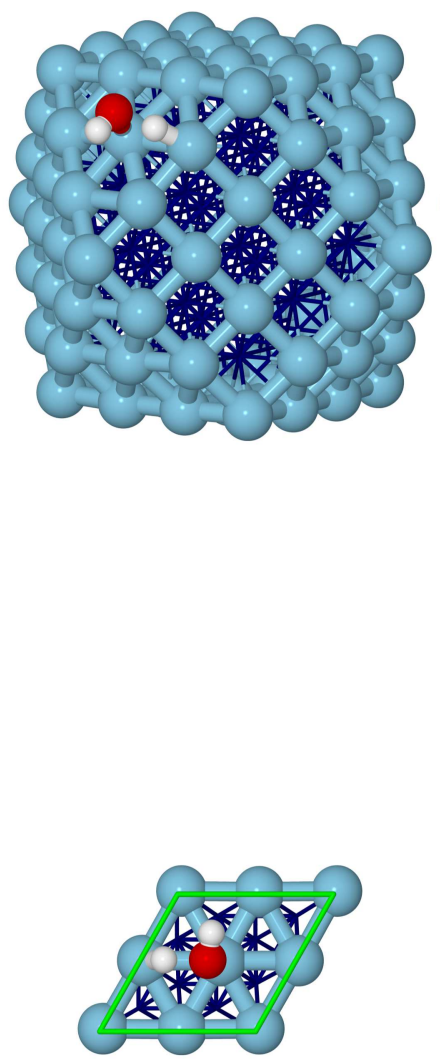

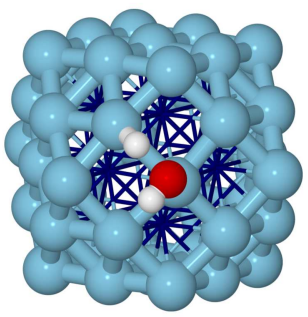

$100 T$
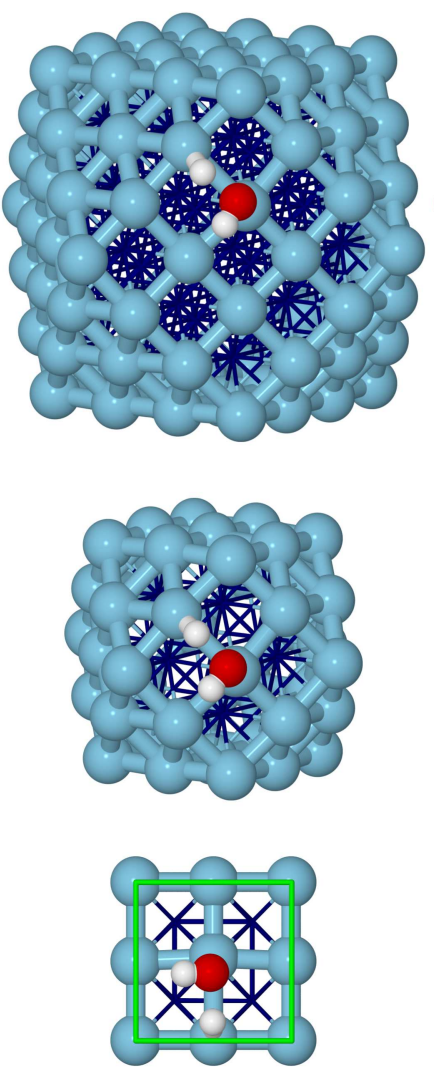

E
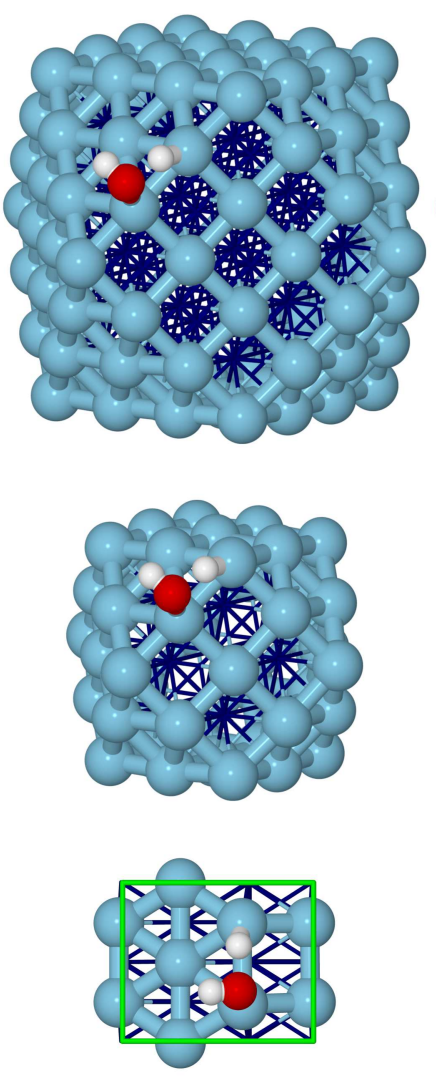

C
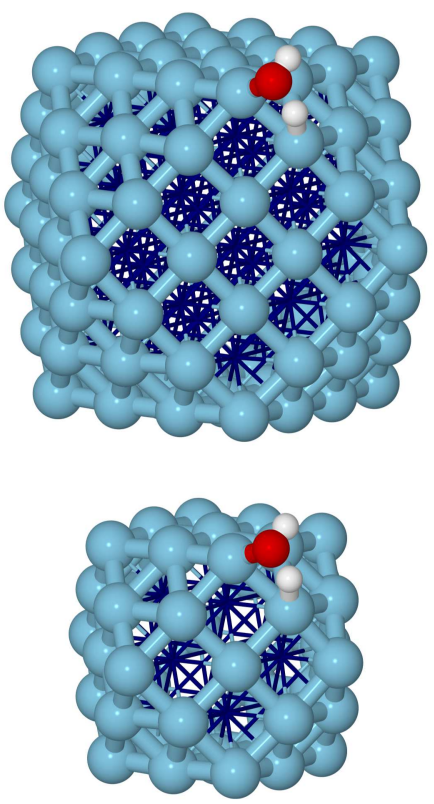

Figure 3: Structures of the transition state of water dissociation for all studied structural models (bulk metal atoms in dark blue, surface metal atoms in light blue, oxygen in red, hydrogen in white, unit cells of the extended surfaces in green). 

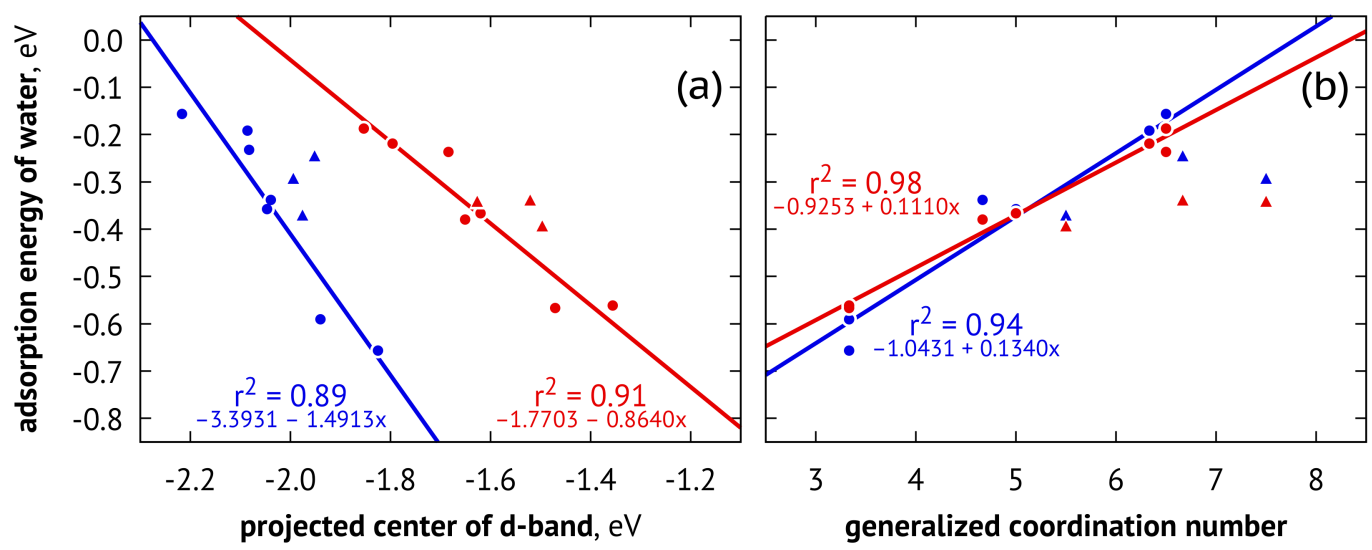

Figure 4: Dependencies of the molecular adsorption of water (a) on the center of the d-band projected onto the corresponding sites and (b) on the generalized coordination numbers (right). Coding: Rh in red, Pt in blue, nanoparticles with circles, extended surfaces with triangles. The trendlines and correlation coefficients are given for the nanoparticle models only.

adsorption energies on the nanoparticle models are consistent with those calculated for the extended surfaces, with an exception for the $111 \mathrm{~T}$ site. The difference may be attributed to the incomplete formation of the (111) facets on the nanoparticles in the selected size regime. Overall, the highly undercoordinated $\mathrm{C}$ sites of the nanoparticles bind water the strongest, as intuitively expected, followed by the E, 100T, and 111T sites in the same order as for the extended surface models.

In the case of the nanoparticle models, the adsorption energies are found to correlate linearly $\left(r^{2}>0.89\right)$ with the d-band center projected onto the corresponding adsorption sites (Figure 4a). Moreover, we observe strong linear dependencies $\left(r^{2}>0.94\right)$ of the adsorption energies on the generalized coordination numbers (Figure 4b), which indicates that the coordination of the surface sites plays a dominant role in determining the strength of interaction. Calle-Vallejo et al. reported similar strong correlations for the truncated octahderal Pt nanoparticles of $0.7-1.7 \mathrm{~nm}$ in size. ${ }^{19-21}$ The data obtained for the extended surface models, however, dropped out from the relationships, suggesting that these models are unable to fully mimic the facets of the cuboctahedral Rh and Pt nanoparticles in the selected size regime. 
As in the case of the adsorption energies, the difference in the calculated transition state energies between $\mathrm{Rh}$ and $\mathrm{Pt}$ is found to be negligibly small (within $0.10 \mathrm{eV}$ ). The most stable transition states on the extended surface models are identified for the E sites on the stepped (211) surfaces, followed by the terrace sites on the (111) and (100) surfaces. The transition state energies calculated for the nanoparticle models were consistent with the extended surface models, again with an exception of the $111 \mathrm{~T}$ site, which is likely due to incomplete formation of the (111) facets on the $\mathrm{Rh}_{147}$ and $\mathrm{Pt}_{147}$ nanoparticles. The most stable transition states on the nanoparticle models were observed at the $\mathrm{C}$ sites, followed by the E, 100T, and $111 \mathrm{~T}$ sites.
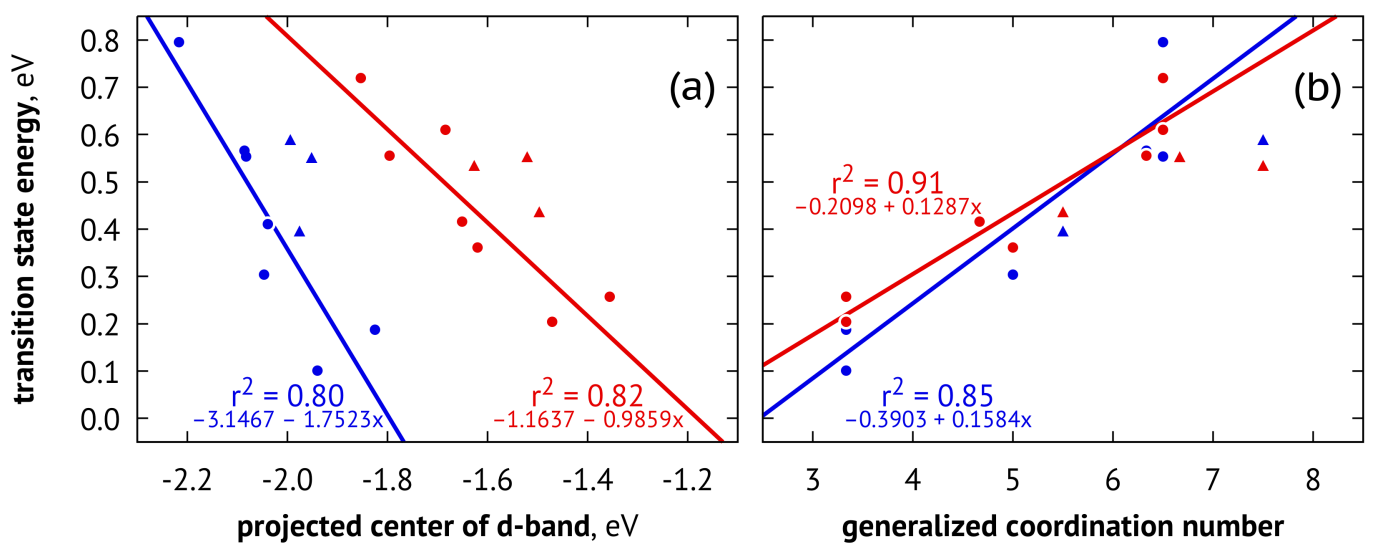

Figure 5: Dependencies of the transition state energy of water activation (a) on the center of the d-band projected onto the corresponding sites and (b) on the generalized coordination numbers. Coding: Rh in red, Pt in blue, nanoparticles with circles, extended surfaces with triangles. The trendlines and correlation coefficients are shown for the nanoparticle models only.

Similarly to the adsorption, the transition state energies calculated for the nanoparticle models demonstrate strong dependencies $\left(r^{2}>0.85\right)$ on the generalized coordination numbers and moderate dependencies $\left(r^{2}>0.80\right)$ on the projected d-band centers (Figure 5). Generalized coordination numbers of the sites occupied by $\mathrm{OH}$ in the transition state complexes were used in the analysis. The observed trends result in the fact that our data show strong $\left(r^{2}>0.85\right)$ Brønsted-Evans-Polanyi-type (BEP) transition state scaling relation- 


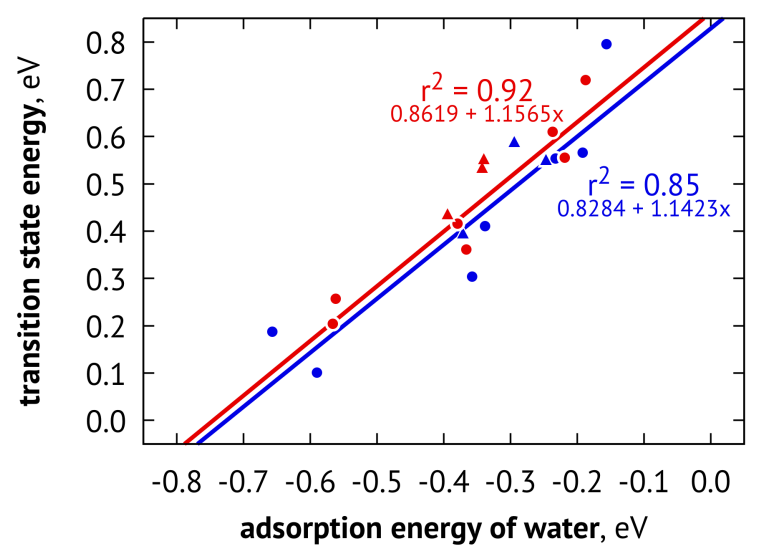

Figure 6: Transition state scaling for the water adsorption and activation. Coding: Rh in red, Pt in blue, nanoparticles with circles, extended surfaces with triangles. The trendlines are shown for the nanoparticle models only.

ships (Figure 6), consistently with the previously reported interpretations. ${ }^{30}$ Nevertheless, these trends are found to be weaker for the extended surface models, further supporting the suggestion that such models are unable to completely describe the electronic and atomic structures of the cuboctahderal $\mathrm{Rh}$ and Pt nanoparticles in the selected size regime. The BEP relationships for water activation on different metal surfaces, including $\mathrm{Rh}$ and Pt, have been previously reported by Fajín et al. ${ }^{31-33}$

The analysis of the complete reaction profiles (Figure 7) revealed that the difference between Rh and Pt metals is hidden behind the structures of the dissociated states of water. As the data in Table 3 show, the $\mathrm{OH}$ and $\mathrm{H}$ species present generally more stable configurations on Rh, making the water activation there thermodynamically more favorable. In terms of energy, the difference was found to be still insignificant $(<0.10 \mathrm{eV})$ in the case of the $\mathrm{Rh}_{55}$ and $\mathrm{Pt}_{55}$ nanoparticles; however, it increased to approximately $0.20 \mathrm{eV}$ for the $\mathrm{M}_{147}$ nanoparticles and further to $>0.30 \mathrm{eV}$ values for the extended surfaces. Among the extended surfaces, the stepped (211) surfaces give the closest values of $\Delta E^{\mathrm{D}}$ to those for the nanoparticles.

The difference between Rh and Pt and the best performance of the (211) extended surfaces may be linked to the different preference of the adsorption sites by the $\mathrm{OH}$ and $\mathrm{H}$ 

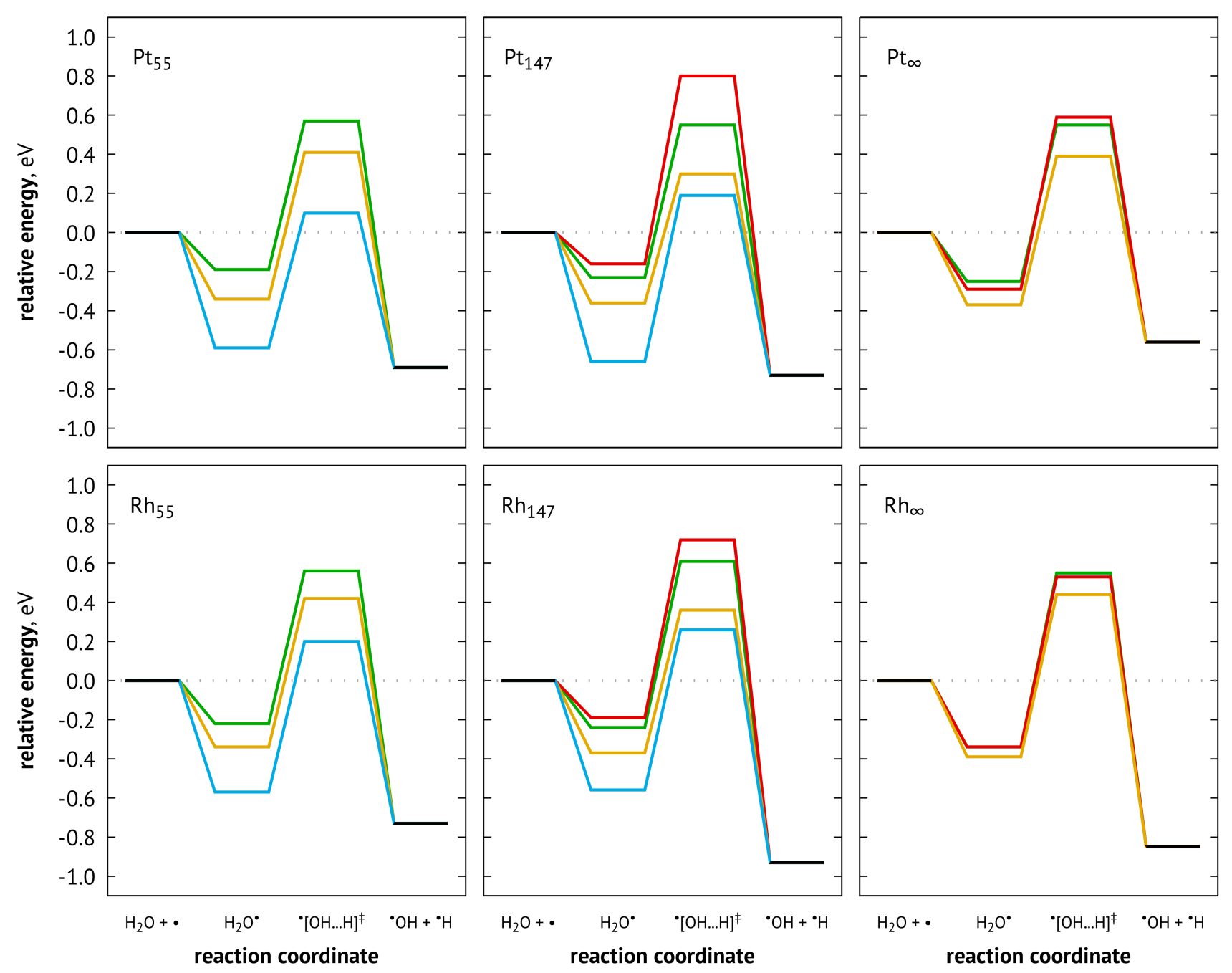

Figure 7: Complete reaction profiles of the adsorption and activation of water on $\mathrm{Rh}$ and $\mathrm{Pt}$ nanoparticles and extended surfaces. Color coding: 111T in red, 100T in green, E in yellow, and $\mathrm{C}$ in blue. See Figure 1 for the notation of the surface sites. 


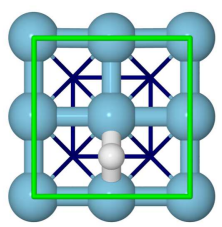

M (100)

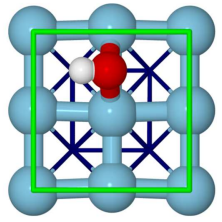

M (100)

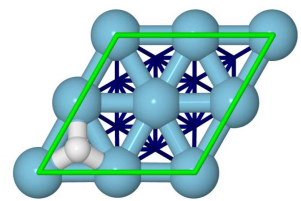

M (111)

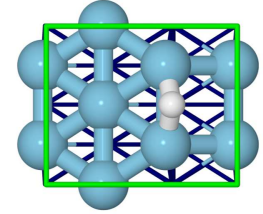

M (211)

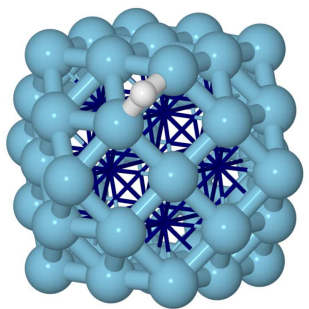

$\mathrm{M}_{55}$

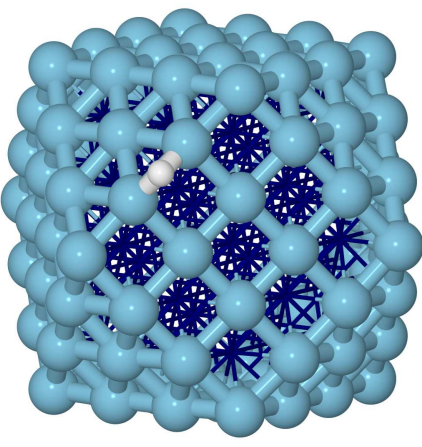

$M_{147}$

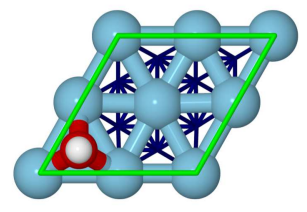

Rh (111)

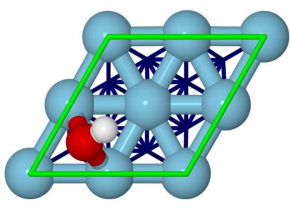

Pt (111)

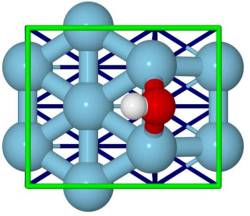

M (211)

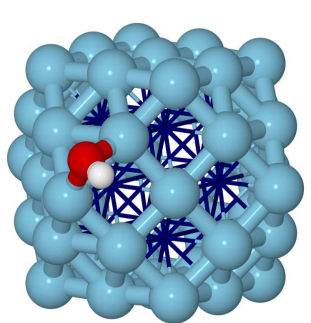

$\mathrm{Rh}_{55}$

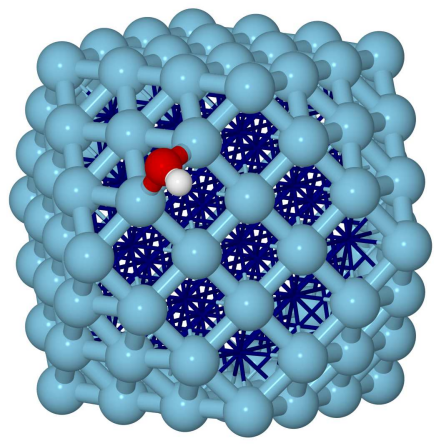

$\mathrm{Rh}_{147}$

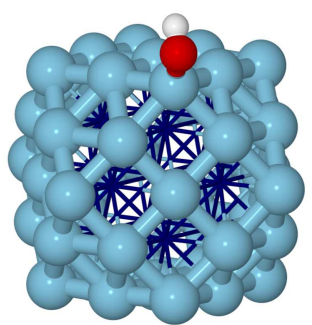

$\mathrm{Pt}_{55}$

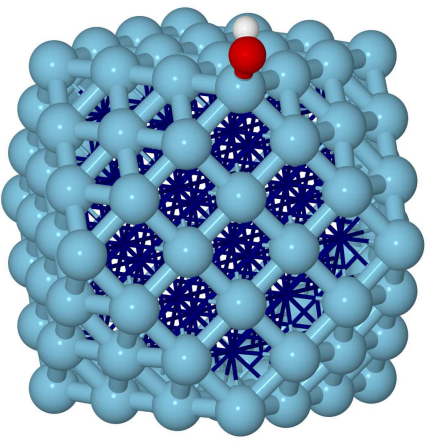

$\mathrm{Pt}_{147}$

Figure 8: Adsorption modes of dissociated water on each studied structural model (bulk metal atoms in dark blue, surface metal atoms in light blue, oxygen in red, hydrogen in white, unit cells of the extended surfaces in green). 
species. In the case of Rh, both hydroxyl and hydrogen favor the E-E bridging sites of the nanoparticles and the corresponding E-E bridging sites at the step edges of the (211) extended surfaces. On the flat surfaces of $\mathrm{Rh}$, both $\mathrm{OH}$ and $\mathrm{H}$ prefer adsorption to the $\mathrm{T}-\mathrm{T}$ bridging sites on the (100) surface and to the $\mathrm{H}$ sites on the (111) surface; however, these configurations are higher in energy. On $\mathrm{Pt}$, the $\mathrm{OH}$ and $\mathrm{H}$ species preferably bind to the $\mathrm{C}$ and $\mathrm{E}-\mathrm{E}$ bridging sites of the nanoparticles, respectively, while on the stepped (211) surface, both species adsorb on the E-E bridging sites, which is the closest approximation of the nanoparticle, considering that there are no $\mathrm{C}$ sites on any of the extended surfaces. High-index extended surfaces might have thus provided necessary kink sites to mimic the corners of the nanoparticles. On the flat surfaces, the hydroxyl favors the $\mathrm{T}-\mathrm{T}$ bridging sites, whereas the hydrogen prefers the T-T bridging site on the (100) surface and the H site on the (111) surface. Figure 8 summarizes the most favorable configurations of dissociated water on each considered structure model. These findings are qualitatively in line with the experimental observations, which suggest that the water activation is less favorable on $\mathrm{Pt}$ due to the low thermodynamical stability of $\mathrm{PtO}_{x} \cdot{ }^{34}$ Moreover, a systematic evaluation of $\mathrm{Pt}$ catalysts supported on reducible oxides has demonstrated that the presence of the support significantly improves performance of Pt in the water-gas shift. ${ }^{35}$

\section{Conclusions}

In summary, we computationally examined and compared water adsorption, activation, and dissociation by the $\mathrm{Rh}$ and $\mathrm{Pt}$ metals. We modeled the metal surfaces using the $\mathrm{M}_{55}$ and $\mathrm{M}_{147}$ cuboctahedral nanoparticles as well as the extended slabs. For the nanoparticle models, the adsorption of water was found to strongly depend on the nature of the surface sites in terms of coordination and the structure of d-band, resulting in strong linear dependencies of the adsorption energies on the considered descriptors, i. e. generalized coordination numbers and the projected centers of the d-band. The transition state energies showed excellent 
scaling relationships with the adsorption energies as well as the strong correlations with the descriptors, enabling prediction of these values at relatively low computational expense. For the extended surface models, the aforementioned relationships were found to be significantly weaker, indicating that such models cannot represent the finite-size effects on the electronic and atomic structures of the cuboctahderal $\mathrm{Rh}$ and $\mathrm{Pt}$ nanoparticles in the chosen size regime $(<2 \mathrm{~nm})$ and the reaction. Nonetheless, the extended surface models do mimic well the energetics of the water activation, and the difference between Rh and Pt metals in their tendencies to adsorb and activate water was overall small $(<0.10 \mathrm{eV})$ using the different structural models. Regardless of the model, the dissociation of water was found to be thermodynamically more favorable on $\mathrm{Rh}(>0.10 \mathrm{eV}$ depending on the model), which is likely due to the different affinity of the $\mathrm{OH}$ and $\mathrm{H}$ species to the $\mathrm{Rh}$ and $\mathrm{Pt}$ metals. Experimentally observed inequalities may be thus linked to the higher stability of the $\mathrm{OH}$ and $\mathrm{H}$ species on $\mathrm{Rh}$ compared to $\mathrm{Pt}$ and/or to the use of the oxide supporting materials, upon interaction with which, metals nanoparticles alter their shapes and, consequently, atomic and electronic structures. Variety of the interface sites, generated thereby, may have different properties compared to the sites in the bare metal nanoparticles.

\section{Acknowledgement}

We gratefully acknowledge the financial support from the Academy of Finland (grant 277222). Electronic structure calculations were made possible through the use of computational resources provided by the CSC - IT Center of Science in Espoo, Finland (http://www.csc.fi/).

\section{References}

(1) Usman, M.; Daud, W. M. A. W.; Abbas, H. F. Dry reforming of methane: Influence of process parameters-A review. Renew. Sustain. Energy Rev. 2015, 45, 710-744. 
(2) Xiong, K.; Yu, W.; Vlachos, D. G.; Chen, J. G. Reaction pathways of biomass-derived oxygenates over metals and carbides: From model surfaces to supported catalysts. Chem. Cat. Chem. 2015, \%, 1402-1421.

(3) Contreras, J. L.; Salmones, J.; Colín-Luna, J. A.; Nuño, L.; Quintava, B.; Córdova, I.; Zeifert, B.; Tapia, C.; Fuentes, G. A. Catalysts for $\mathrm{H}_{2}$ production using the ethanol steam reforming (a review). Int. J. Hydrogen Energy 2014, 39, 18835-18853.

(4) Mattos, L. V.; Jacobs, G.; Davis, B. H.; Noronha, F. B. Production of hydrogen from ethanol: Review of reaction mechanism and catalyst deactivation. Chem. Rev. 2012, 112, 4094-4123.

(5) Yu, W.; Porosoff, M. D.; Chen, J. G. Review of Pt-based bimetallic catalysis: From model surfaces to supported catalysts. Chem. Rev. 2012, 112, 5780-5817.

(6) Ratnasamy, C.; Wagner, J. P. Water gas shift catalysis. Catal. Rev. 2009, 51, 325-440.

(7) Kalamaras, C. M.; Gonzales, I. D.; Navarro, R. M.; Fierro, J. L. G.; Efstathiou, A. M. Effects of reaction temperature and support composition on the mechanism of water-gas shift reaction over supported-Pt catalysts. J. Phys. Chem. C 2011, 115, 11595-11610.

(8) Rodriguez, J. A.; Liu, P.; Hrbek, J.; Evans, J.; Pérez, M. Water gas shift reaction on $\mathrm{Cu}$ and $\mathrm{Au}$ nanoparticles supported on $\mathrm{CeO}_{2}(111)$ and $\mathrm{ZnO}(000 \overline{1})$ : Intrinsic activity and importance of support interactions. Angew. Chem. Int. Ed. 2007, 46, 1329-1332.

(9) Clay, J. P.; Greeley, J. P.; Ribeiro, F. H.; Delgass, W. N.; Schneider, W. F. DFT comparison of intrinsic WGS kinetics over Pd and Pt. J. Catal. 2014, 320, 106-117.

(10) Peköz, R.; Wörger, S.; Ghiringhelli, L. M.; Donadio, D. Trends in the adsorption and dissociation of water clusters on flat and stepped metallic surfaces. J. Phys. Chem. C 2014, 118, 29990-29998. 
(11) Trinchero, A.; Hellman, A.; Grönbeck, H. Methane oxidation over Pd and Pt studied by DFT and kinetic modeling. Surf. Sci. 2013, 616, 206-213.

(12) Fajín, J. L. C.; Bruix, A.; Cordeiro, M. N. D. S.; Gomes, J. R. B.; Illas, F. Density functional theory model study of size and structure effects on water dissociation by platinum nanoparticles. J. Chem. Phys. 2012, 137, 034701.

(13) Lin, C. H.; Chen, C. L.; Wang, J. H. Mechanistic studies of water-gas-shift reaction on transition metals. J. Phys. Chem. C 2011, 115, 18582-18588.

(14) German, E. D.; Sheintuch, M. Quantum effects in the kinetics of $\mathrm{H}_{2} \mathrm{O}$ dissociative adsorption on $\mathrm{Pt}(111), \mathrm{Cu}(111), \mathrm{Rh}(111)$, and $\mathrm{Ni}(111)$. J. Phys. Chem. C 2010, 114, 3089-3097.

(15) Phatak, A. A.; Delgass, W. N.; Ribeiro, F. H.; Schneider, W. F. Density functional theory comparison of water dissociation steps on $\mathrm{Cu}, \mathrm{Au}, \mathrm{Ni}, \mathrm{Pd}$, and Pt. J. Phys. Chem. C 2009, 113, 7269-7276.

(16) Grabow, L. C.; Gokhale, A. A.; Evans, S. T.; Dumesic, J. A.; Mavrikakis, M. Mechanism of the water gas shift reaction on Pt: First principles, experiments, and microkinetic modeling. J. Phys. Chem. C 2008, 112, 4608-4617.

(17) Wang, G. C.; Tao, S. X.; Bu, X. H. A systematic theoretical study of water dissociation on clean and oxygen-preadsorbed transition metals. J. Catal. 2006, 244, 10-16.

(18) Vassilev, P.; van Santen, R. A.; Koper, M. T. M. Ab initio studies of a water layer at transition metal surfaces. J. Chem. Phys. 2005, 122, 054701.

(19) Calle-Vallejo, F.; Martínez, J. I.; García-Lastra, J. M.; Sautet, P.; Loffreda, D. Fast prediction of adsorption properties for platinum nanocatalysts with generalized coordination numbers. Angew. Chem. Int. Ed. 2014, 53, 8316-8319. 
(20) Calle-Vallejo, F.; Sautet, P.; Loffreda, D. Understanding adsorption-induced effects on platinum nanoparticles: An energy-decomposition analysis. J. Phys. Chem. Lett. 2014, $5,3120-3124$.

(21) Calle-Vallejo, F.; Tymoczko, J.; Colic, V.; Vu, Q. H.; Pohl, M. D.; Morgenstern, K.; Loffreda, D.; Sautet, P.; Schuhmann, W.; Bandarenka, A. S. Finding optimal surface sites on heterogeneous catalysts by counting nearest neighbors. Science 2015, 350, $185-189$.

(22) Mortensen, J. J.; Hansen, L. B.; Jacobsen, K. W. Real-space grid implementation of the projector augmented wave method. Phys. Rev. B 2005, 71, 035109.

(23) Enkovaara, J.; C., R.; Mortensen, J. J.; Chen, J.; Dułak, M.; Ferrighi, L.; Gavnholt, J.; Glinsvad, C.; Haikola, V.; Hansen, H. A. et al. Electronic structure calculations with GPAW: A real-space implementation of the projector augmented-wave method. J. Phys.: Condens. Matter 2010, 22, 253202.

(24) Perdew, J. P.; Burke, K.; Ernzerhof, M. Generalized gradient approximation made simple. Phys. Rev. Lett. 1996, r7, 3865-3868.

(25) Perdew, J. P.; Burke, K.; Ernzerhof, M. Generalized gradient approximation made simple [Phys. Rev. Lett. 77, 3865 (1996)]. Phys. Rev. Lett. 1997, 78, 1396-1396.

(26) Bahn, S. R.; Jacobsen, K. W. An object-oriented scripting interface to a legacy electronic structure code. Comput. Sci. Eng. 2002, 4, 56-66.

(27) Crystalium: An exploration of the surfaces and Wulff shapes of the elements. http: //crystalium.materialsvirtuallab.org, accessed: 2017-01-31.

(28) Tran, R.; Xu, Z.; Radhakrishnan, B.; Winston, D.; Sun, W.; Persson, K. A.; Ong, S. P. Surface energies of elemental crystals. Sci. Data 2016, 3, 160080. 
(29) Fajín, J. L. C.; Cordeiro, M. N. D. S.; Gomes, J. R. B. Density functional theory study of the water dissociation on platinum surfaces: General trends. J. Phys. Chem. A 2014, $118,5832-5840$.

(30) Calle-Vallejo, F.; Martínez, J. I.; García-Lastra, J. M.; Rossmeisl, J.; Koper, M. T. M. Physical and chemical nature of the scaling relations between adsorption energies of atoms on metal surfaces. Phys. Rev. Lett. 2012, 108, 116103.

(31) Fajín, J. L. C.; Viñ, F.; Cordeiro, M. N. D. S.; Illas, F.; Gomes, J. R. B. Effect of the exchange-correlation potential on the transferability of Brønsted-Evans-Polanyi relationships in heterogeneous catalysis. J. Chem. Theory Comput 2016, 12, 21212126.

(32) Fajín, J. L. C.; Cordeiro, M. N. D. S.; Illas, F.; Gomes, J. R. B. Generalized BrønstedEvans-Polanyi relationships and descriptors for $\mathrm{O}-\mathrm{H}$ bond cleavage of organic molecules on transition metal surfaces. J. Catal. 2014, 313, 24-33.

(33) Fajín, J. L. C.; Cordeiro, M. N. D. S.; Illas, F.; Gomes, J. R. B. Descriptors controlling the catalytic activity of metallic surfaces toward water splitting. J. Catal. 2010, 276, $92-100$.

(34) Takanabe, K.; Aika, K.-i.; Seshan, K.; Lefferts, L. Sustainable hydrogen from bio-oilSteam reforming of acetic acid as a model oxygenate. J. Catal. 2004, 227, 101-108.

(35) Azzam, K. G.; Babich, I. V.; Seshan, K.; Lefferts, L. Bifunctional catalysts for singlestate water-gas shift reaction in fuel cell applications. Part 1. Effect of the support on the reaction sequence. J. Catal. 2007, 251, 153-162. 


\section{TOC Graphic}

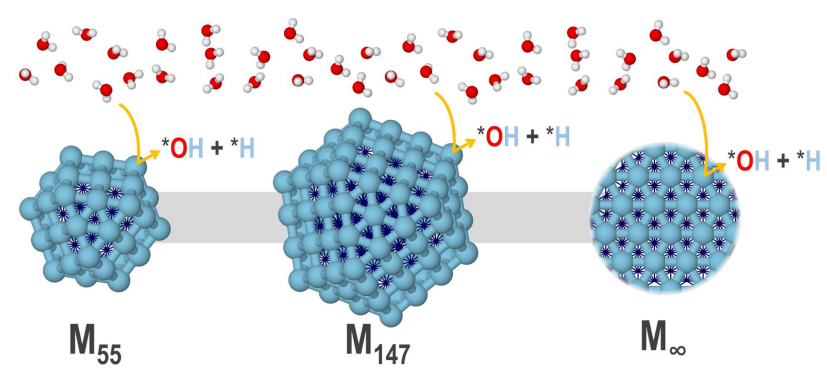

16

17

18

19

20

21

22

23

24

25

26

27

28

29

30

31

32

33

34

35

36

37

38

39

40

41

42

43

44

45

46

47

48

49

50

51

52

53

54

55

56

57

58

59

60 